\title{
Tumor PD-L1 Expression and Clinical Outcomes in Advanced-stage Non-Small Cell Lung Cancer Patients Treated with Nivolumab or Pembrolizumab: Real-World Data in Taiwan
}

\author{
Shu-Yung Lin ${ }^{1,2}$, Ching-Yao Yang ${ }^{3,4 \bowtie}$, Bin-Chi Liao ${ }^{2,5}$, Chao-Chi Ho ${ }^{4}$, Wei-Yu Liao ${ }^{4}$, Kuan-Yu Chen, \\ Tzu-Hsiu Tsai ${ }^{4}$, Chia-Lin Hsu ${ }^{4}$, Wei-Hsun Hsu ${ }^{5}$, Kang-Yi Su ${ }^{6}$, Yih-Leong Chang ${ }^{3,7}$, Jih-Hsiang Lee ${ }^{5}$, \\ Chia-Chi Lin 5 , Jin-Yuan Shih", James Chih-Hsin Yang5, Chong-Jen Yu ${ }^{4}$
}

1. Department of Internal Medicine, National Taiwan University Hospital Jin-Shan Branch, New Taipei City, Taiwan

2. Graduate Institute of Clinical Medicine, National Taiwan University College of Medicine, Taipei City, Taiwan

3. Graduate Institute of Pathology, National Taiwan University College of Medicine, Taipei City, Taiwan

4. Department of Internal Medicine, National Taiwan University Hospital, Taipei City, Taiwan

5. Department of Oncology, National Taiwan University Hospital, Taipei City, Taiwan

6. Department of Clinical Laboratory Sciences and Medical Biotechnology, National Taiwan University College of Medicine, Taipei City, Taiwan

7. Department of Pathology, National Taiwan University Hospital and National Taiwan University College of Medicine, Taipei City, Taiwan

$\square$ Corresponding author: Ching-Yao Yang MD., Department of Internal Medicine, National Taiwan University Hospital, No. 7, Chung-Shan S. Rd., Taipei 100, Taiwan. Tel: 886-2-2312-3456; E-mail: chingyao.yang@gmail.com

(c) Ivyspring International Publisher. This is an open access article distributed under the terms of the Creative Commons Attribution (CC BY-NC) license (https://creativecommons.org/licenses/by-nc/4.0/). See http://ivyspring.com/terms for full terms and conditions.

Received: 2018.01.16; Accepted: 2018.02.26; Published: 2018.04.19

\begin{abstract}
Background: Immunotherapy that targets programmed death protein-1 (PD-1) provides improved treatment efficacy and survival in patients with metastatic non-small cell lung cancer (NSCLC), especially those with high tumor expression of PD-L1. However, data on this treatment are mostly from clinical trials enrolling highly selected patients. The real-world experience of anti-PD-1 treatment and the usefulness of tumor PD-L1 expression in prediction of treatment response are largely unknown.

Methods: We retrospectively reviewed patients with stage IIIB/ IV NSCLC who received monotherapy with nivolumab or pembrolizumab, and evaluated response using RECIST 1.1 criteria. Factors associated with treatment response, progression free survival (PFS), and overall survival (OS) were determined.

Results: Seventy-four NSCLC patients out of 116 examined patients were included, most of whom had adenocarcinoma $(48 / 74,64.9 \%)$ and received immunotherapy as a third-line or subsequent treatment $(51 / 74$, 68.9\%). The median PFS and OS were 1.8 and 7.9 months, respectively. The objective response rate was $32 \%$, but only 47 of 74 patients were evaluable. Through multivariate analysis, epidermal growth factor receptor (EGFR) mutation was independently associated with a poor treatment response. Good performance status $(E C O G \leq 1)$ and smoking were independently associated with better PFS and OS. Data on tumor PD-L1 expression were available in 43 patients (58\%); higher PD-L1 expression correlated with better treatment response and longer PFS. Severe treatment-related adverse events were uncommon.

Conclusion: The efficacy and safety of anti-PD-1 medications for advanced NSCLC were comparable in real-world and clinical settings, except in those with poor ECOG scores. Prediction of treatment response from tumor PD-LI expression seemed practical.
\end{abstract}

Key words: Immunotherapy, Non-small cell lung cancer, Programmed death 1, Real-world, Survival

\section{Introduction}

The treatment of advanced non-small cell lung cancer (NSCLC) has evolved from platinum-based chemotherapy to molecular targeted therapy and immunotherapy.[1] There is currently great interest in the use of immune checkpoint inhibitors that target the programmed death-ligand 1 (PD-L1)/PD-1 axis. 
Many phase 3 clinical trials have confirmed anti-PD-1 treatments in advanced NSCLC patients, either as a second-line[2-4] or first-line treatment[5] provide improved efficacy and survival. Compared with second-line docetaxel chemotherapy, treatment with nivolumab or pembrolizumab (anti-PD-1 monoclonal antibodies) provided a better objective response rate (ORR) and overall survival (OS).[2-4] The ORR of these agents range from $18-20 \%$ and the OS from 9.2 to 12.4 months. When used as a first-line treatment, pembrolizumab led to a significantly better ORR, progression free survival (PFS), and OS than platinum-based chemotherapy among patients with PD-L1 expression over 50\%.[5] Patients with high tumor expression of PD-L1 seemed more likely to benefit from anti-PD-1 treatment, albeit responses were seen even in patients with low or no PD-L1 expression.[2, 4-6]

Previous studies of anti-PD-1 treatment were almost all based on clinical trials, with enrollment of highly selected patients. However, 'real-world' patients with NSCLC are more heterogeneous than those studied in clinical trials, in that they typically have more diverse performance status, co-morbidities, organ dysfunctions, and may also present with unstable brain metastasis. Only a few small cohort studies reported the real-world efficacy of anti-PD-1 treatment, and these studies examined the effect of nivolumab in Westerners.[7, 8] NSCLC patients from East Asia are mostly non-smokers and have a higher prevalence of epidermal growth factor receptor (EGFR) mutations; [9] the effect of anti-PD-1 agents in these patients in real-world settings are unknown. There is also limited real-world experience regarding the relationship of tumor PD-L1 expression in NSCLC and treatment response.

The rapid adoption of immunotherapy for treatment of lung cancer worldwide[10] motivated us to conduct a retrospective study of the real-world experience of anti-PD-1 treatment in an Eastern Asian cohort with advanced-stage NSCLC. We examined the effect of different clinical and demographic characteristics, as well as tumor PD-L1 expression on treatment response and clinical outcomes.

\section{Methods}

\section{Patient demographics and outcome measurements}

The records of all patients who received monotherapy consisting of immunotherapy with nivolumab or pembrolizumab in the tertiary referral center of National Taiwan University Hospital (NTUH) were reviewed. Enrolled patients had pathologically confirmed NSCLC from April 2015 to
August 2017. This study was approved by the Research Ethics Committee of NTUH (201705049 RIND).

Demographics, smoking status, Eastern Cooperative Oncology Group (ECOG) status, and previous therapies for NSCLC were recorded. Mutations of EGFR, Kirsten rat sarcoma virus oncogene homolog (KRAS), and anaplastic lymphoma kinase (ALK) from tumor samples were recorded if available. Smoker was defined as lifetime use of more than 100 cigarettes.[11] Follow up data was acquired by review of medical charts or by direct telephone contact. All patients received radiological assessment of tumor response by computed tomography (CT) every 4 to 12 weeks. The outcome measurements were response to immunotherapy, PFS, and OS. PFS was defined as the time from the start of treatment to disease progression or death. OS was defined as time from the start of treatment to death from any cause. The response to therapy was assessed using Response Evaluation Criteria in Solid Tumors (RECIST) version 1.1. Response to treatment was defined as best overall response during entire follow up period. Evaluable patients were those who received an immunotherapy agent for at least 8 weeks and took subsequent $\mathrm{CT}$ at least once for response evaluation. Adverse events from immunotherapy were reported based on review of the medical records. Patients who received chemotherapy after failure of immunotherapy were followed to determine the second PFS (PFS2), defined as the time from initiating subsequent therapy to disease progression or death. The cut-off time for analysis was August 30, 2017.

\section{Mutational analysis of EGFR and KRAS}

Testing for genetic mutations in EGFR and KRAS was performed routinely in an ISO15189-certificated central lab, as previously reported.[12, 13] Briefly, genomic DNA was extracted using the QIAmp DNA Minikit (QIAGEN, CA) according to the manufacturer's instructions, and mutations were identified using the MassARRAY system (Agena, CA) based on the users' manual. Extracted DNA was subjected to serial biochemical reactions, consisting of 40 cycles of PCR, shrimp alkaline phosphatase (SAP) treatment, and 200 cycles of a signal nucleotide extension reaction. After cleaning using SpectroCLEAN resin, samples were loaded onto the matrix of a SpectroCHIP by Nanodispenser (Matrix), and then analyzed by Bruker Autoflex MALDI-TOF MS. Data were collected and analyzed using Typer4 software (Agena Bioscience, San Diego, CA). 
116 patients with diagnosis of lung cancer receiving anti-PD1 treatment

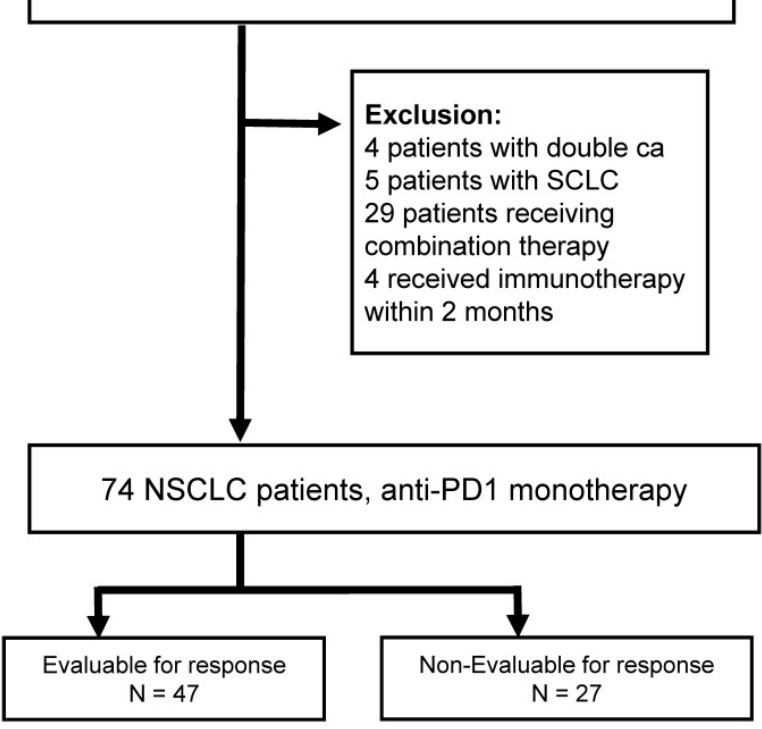

Figure 1: CONSORT diagram of the screened NSCLC patients $(n=116)$. Abbreviations: NSCLC: non-small cell lung cancer, PD-1: programmed death 1, SCLC: small cell lung cancer.

\section{Immunohistochemical staining and scoring for PD-LI}

Immunohistochemical detection of tumor PD-L1 expression was determined using the PD-L1 Clone 22C3 kit (pharmDx) and the Automated Link 48 platform (Dako, Carpinteria, CA). The percentage of tumor cells with PD-L1 expression (positive membrane staining) was determined by counting at least 100 viable cells. This analysis was provided by Dr. Yih-Leong Chang and other pathologists in the National Taiwan University Hospital.

\section{Statistical Analysis}

The Kaplan-Meier method was used to estimate OS and PFS, and the log-rank test was used to determine the significance of differences in these parameters. Logistic regression was used to identify factors associated with objective response (according to RECIST 1.1), and a Cox proportional hazards model was used for multivariable survival analysis, in which the adjusted variables were age, sex, smoking, and variables with $p$-values below 0.1 in the univariate analysis. Statistical analysis was carried out using SPSS version 22 (IBM Software, Armonk).

\section{Results}

\section{Patient demographic and clinical characteristics}

We retrospectively examined the records of 116 patients with lung cancer receiving anti-PD1 treatment, 74 of whom received monotherapy with pembrolizumab or nivolumab between April 2015 and August 2017. The median follow up time was 12.4 months. We excluded 42 cases, mainly due to use of combination therapy (Figure 1). All 74 included patients received standard dosages of nivolumab (3 $\mathrm{mg} / \mathrm{kg}$ every 2 weeks) or pembrolizumab $(2 \mathrm{mg} / \mathrm{kg}$ every 3 weeks).

Analysis of patient demographic and clinical characteristics indicates that most patients received extensive treatment before immunotherapy, and nearly $70 \%$ received immunotherapy as a third-line or subsequent treatment (Table 1). Adenocarcinoma was the most common histology, followed by squamous cell carcinoma. Nearly half of the population had ECOG status of 2 or more. Among patients with known EGFR mutation status $(\mathrm{n}=61)$, mutations were present in 25 patients (25/61, 41\%); among those with known KRAS mutation status $(n=40)$, mutations were in 10 patients $(10 / 40,25 \%)$. None of the tumors had ALK overexpression based on IHC.

Table 1: Demographic and clinical characteristics of NSCLC patients $(n=74)$.

\begin{tabular}{lll}
\hline Characteristic & $\mathrm{n}$ & \\
\hline Age, median (range) & 62.1 & $34.1-86.7$ \\
Male, $\%$ & 43 & $58.1 \%$ \\
Stage IIIB/IV & $2 / 72$ & \\
Smokers, $\%$ & $31 / 71$ & $52.7 \%$ \\
Histology, $\%$ & & \\
Adenocarcinoma & 48 & $64.9 \%$ \\
Squamous cell carcinoma & 14 & $18.9 \%$ \\
Pleomorphic carcinoma & 4 & $5.4 \%$ \\
Lymphoepithelioma-like carcinoma & 6 & $8.1 \%$ \\
Poorly differentiated carcinoma & 2 & $2.7 \%$ \\
ECOG $\geq 2$ before anti-PD-1 treatment & 36 & $48.6 \%$ \\
Radiotherapy before anti-PD-1 treatment & 47 & $63.5 \%$ \\
Nivolumab/Pembrolizumab & $24 / 50$ & \\
Anti-PD-1 as $\geq 3 L$ treatment & 51 & $68.9 \%$ \\
Previous lines of treatment, median (range) & 3 & $0-10$ \\
Brain metastasis, \% & 33 & $44.6 \%$ \\
EGFR mutation, $\%$ & $25 / 61$ & $41 \%$ \\
KRAS mutation. \% & $10 / 40$ & $25 \%$ \\
PD-L1 status, $\%$ & & \\
$\geq 50 \%$ & $17 / 43$ & $39.5 \%$ \\
1-50\% & $16 / 43$ & $37.2 \%$ \\
$<1 \%$ & $10 / 43$ & $23.3 \%$ \\
\hline Abbrev
\end{tabular}

Abbreviations: 3L: third line. ECOG: Eastern Cooperative Oncology Group performance status. EGFR: epidermal growth factor receptor. KRAS: Kirsten rat sarcoma virus oncogene homolog.

\section{Prediction of treatment response}

We analyzed the response of all evaluable patients and of patients grouped by PD-L1 status (Figure 2A and 2B). The ORR was 32\% among all evaluable patients $(n=47)$. Patients with higher expression of tumor PD-L1 had a better ORR, although this was not significant $(p=0.195)$. As shown in Table S2, wild type EGFR and KRAS mutant patients might had better treatment response. Among the limited number of patients with both EGFR and 


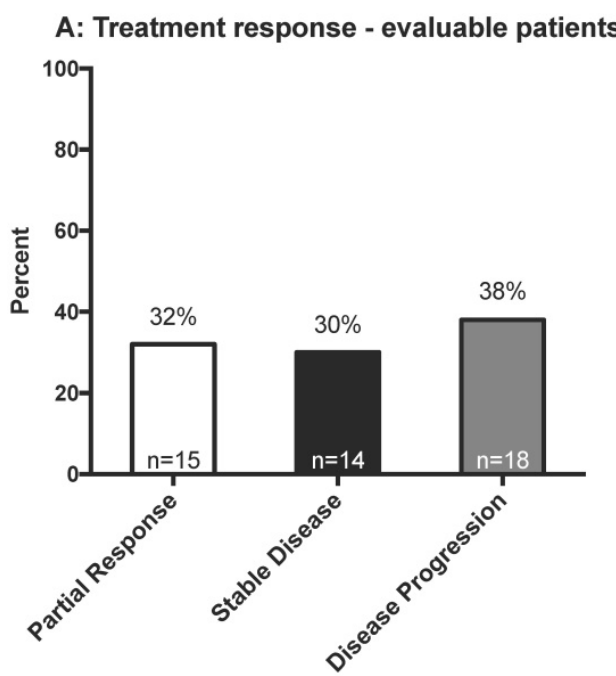

B: Treatment resposne grouped by known PD-L1 status

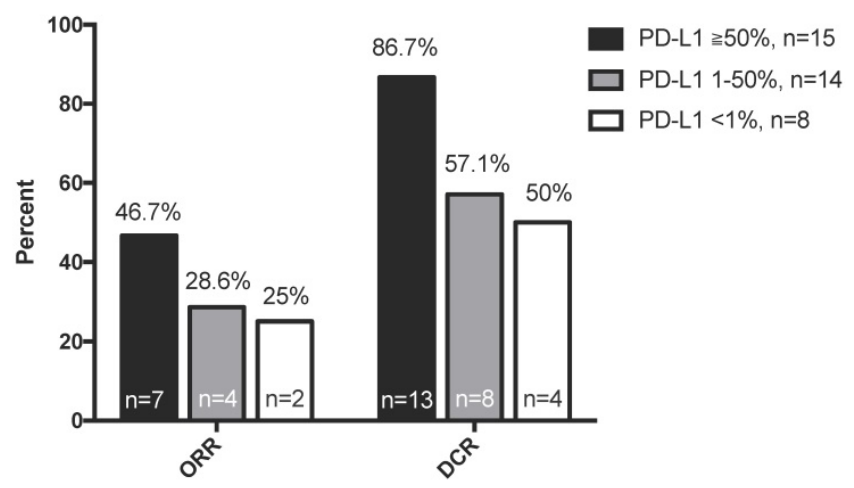

Figure 2: A: Best treatment response of all evaluable patients $(n=47)$; B: Best treatment response according to PD-L1 status $(n=43)$. Abbreviations: $D C R$, disease control rate; ORR, objective response rate; PD-LI, programmed death-ligand 1 .

PD-L1 status, better treatment response was observed with high PD-L1 expression among EGFR wild type patients, but the trend was not observed in EGFR mutant patients (Table S2).

Logistic regression analysis of the factors associated with objective treatment response showed that only EGFR mutation was significantly and independently associated with lower treatment response $(\mathrm{OR}=0.09,95 \% \mathrm{CI}=0.01-0.93, \mathrm{p}=0.043)$ (Table $2)$. However, a large proportion of patients $(27 / 74$, $36.5 \%$ ) could not be evaluated for treatment response. These patients mostly had poor performance status (ECOG $>3$ in 24/27, 89\%) and died shortly after immunotherapy (Table S1 and Figure S1).

\section{Clinical outcomes of all patients and potential predictors}

Figures $3 \mathrm{~A}$ and $\mathrm{B}$ show the PFS and OS of all patients, including those who were non-evaluable. Multivariate analysis showed that smoking was significantly and independently associated with a more favorable PFS $(\mathrm{HR}=0.45,95 \%$ CI $0.22-0.92$, $p=0.029)$ and $\mathrm{OS} \quad(\mathrm{HR}=0.44,95 \% \quad \mathrm{CI}=0.21-0.91$, $p=0.026$ ), and that an ECOG score of 2 or more was independently and significantly associated with poor PFS (HR=9.53, 95\% CI=4.23-21.51, $p<0.001)$ and OS $(\mathrm{HR}=14.72,95 \% \mathrm{CI}=6.01-36.05, \mathrm{p}<0.001)$ (Table 3).

Stratification of PFS and OS by ECOG score indicated that patients with ECOG scores of $0-1$ who received anti-PD-1 treatment had a median PFS of 4.8 months, while this group did not attain a median OS (Figures 3C and D). Tumor PD-L1 expression was only available in 43 of 74 patients (58\%). The median PFS of patients with $50 \%$ or more PD-L1-positive cells was 8.0 months, significantly longer than those with 1-49\% PD-L1-positive cells (2.0 months) and those with fewer than 1\% PD-L1-positive cells (2.4 months) $(p=0.03)$ (Figure 3E). There were no differences in OS after stratification by PD-L1 expression (Figure 3F). Patients with wild type EGFR had better PFS compared with those with mutant EGFR (1.3 vs 2.8 months), but there was no difference in OS. There was no difference regarding survival among KRAS wild type or mutant patients (Figure S3).

Table 2: Factors associated with treatment response.

\begin{tabular}{lllll}
\hline & Unadjusted & & Adjusted \\
\hline Variable & OR (95\%CI) & p & aOR (95\%CI) & p \\
\hline Age $\geq 65$ years & $1.90(0.55-6.59)$ & 0.309 & $2.22(0.44-11.28)$ & 0.336 \\
Male $v$ s. Female & $1.05(0.29-3.84)$ & 0.944 & - & - \\
Smoker & $1.32(0.38-4.59)$ & 0.659 & $0.22(0.03-1.87)$ & 0.165 \\
ECOG $\geq 2$ vs. $<2$ & $0.34(0.06-1.79)$ & 0.202 & - & - \\
Brain metastasis & $0.67(0.19-2.31)$ & 0.523 & - & - \\
Radiotherapy before IO & $0.60(0.17-2.09)$ & 0.421 & - & - \\
IO treatment $\geq 3^{\text {rd }}$ line & $0.34(0.1-1.22)$ & 0.099 & $0.25(0.05-1.32)$ & 0.103 \\
Pembrolizumab vs. & $1.56(0.43-5.6)$ & 0.499 & - & - \\
Nivolumab & & & & - \\
Steroid use & $0.83(0.14-4.87)$ & 0.837 & - & - \\
Serious infection $\neq$ & $1.96(0.44-8.71)$ & 0.375 & - & $\mathbf{0 . 0 4 3}$ \\
EGFR mutation & $0.17(0.03-0.94)$ & 0.042 & $\mathbf{0 . 0 9 ( 0 . 0 1 - 0 . 9 3 )}$ & - \\
KRAS mutation & $2.25(0.34-14.69)$ & 0.397 & - & - \\
\hline
\end{tabular}

*Defined as prednisolone use of $10 \mathrm{mg} /$ day (or equivalent) for more than 2 weeks during immunotherapy.

$\neq$ Requiring intravenous antibiotics.

Abbreviations here and below: IO, Immunotherapy; ECOG, Eastern Cooperative Oncology Group performance status.

Gender was excluded from the multivariate analysis due to its high correlation with smoking (Pearson's correlation: $p<0.001$ )

\section{Treatment-related adverse effects}

Abnormal thyroid function was the most common adverse effect $(5 / 74,6.5 \%)$, in that 3 patients developed hypothyroidism and 2 developed hyperthyroidism. The most serious adverse event was grade-4 pneumonitis with subsequent mortality, which occurred in 3 patients (3/74, 3.8\%). Two patients had serositis, 1 had a skin rash, 1 had grade- 2 pneumonitis, and 1 had adrenal insufficiency. 
Table 3: Cox-proportional hazards model of factors associated with progression free survival and overall survival.

\begin{tabular}{|c|c|c|c|c|c|c|c|c|}
\hline & \multicolumn{4}{|c|}{ Progression free survival } & \multicolumn{4}{|l|}{ Overall survival } \\
\hline & Univariate & & Multivariate & & Univariate & & Multivariate & \\
\hline Variable & HR $(95 \% C I)$ & p & HR $(95 \% C I)$ & $\mathrm{p}$ & HR $(95 \% C I)$ & $\mathbf{P}$ & HR $(95 \% C I)$ & p \\
\hline Age $\geq 65$ years & $1.13(0.68-1.88)$ & 0.636 & $0.59(0.32-1.08)$ & 0.087 & $1.32(0.70-2.49)$ & 0.398 & $0.70(0.35-1.42)$ & 0.326 \\
\hline Male vs. Female & $0.76(0.45-1.26)$ & 0.282 & - & - & $0.71(0.37-1.34)$ & 0.285 & - & - \\
\hline Smoker & $0.67(0.41-1.12)$ & 0.128 & $0.45(0.22-0.92)$ & 0.029 & $0.72(0.38-1.37)$ & 0.318 & $0.44(0.21-0.91)$ & 0.026 \\
\hline $\mathrm{ECOG} \geq 2$ vs. $<2$ & $4.76(2.73-8.30)$ & $<0.001$ & $9.13(4.08-20.45)$ & $<0.001$ & $11.14(4.87-25.46)$ & $<0.001$ & $14.72(6.01-36.05)$ & $<0.001$ \\
\hline Brain metastasis & $1.62(0.97-2.73)$ & 0.067 & $0.76(0.38-1.53)$ & 0.445 & $1.37(0.72-2.59)$ & 0.339 & - & - \\
\hline Radiotherapy before IO & $1.37(0.79-2.39)$ & 0.259 & - & - & $1.53(0.74-3.16)$ & 0.247 & - & - \\
\hline IO treatment $\geq 3^{\text {rd }}$ line & $1.26(0.70-2.27)$ & 0.437 & - & - & $1.09(0.52-2.26)$ & 0.822 & - & - \\
\hline Pembrolizumab vs. Nivolumab & $1.28(0.75-2.19)$ & 0.371 & - & - & $1.70(0.83-3.5)$ & 0.147 & - & - \\
\hline Steroid use ${ }^{*}$ & $1.24(0.61-2.53)$ & 0.551 & - & - & $1.61(0.71-3.67)$ & 0.257 & - & - \\
\hline Serious infection $\neq$ & $1.20(0.63-2.27)$ & 0.577 & - & - & $1.40(0.63-3.07)$ & 0.406 & - & - \\
\hline EGFR mutation & $2.00(1.11-3.62)$ & 0.022 & $1.26(0.61-2.60)$ & 0.534 & $1.07(0.50-2.26)$ & 0.867 & - & - \\
\hline KRAS mutation & $0.73(0.32-1.66)$ & 0.457 & - & - & $1.28(0.49-3.38)$ & 0.614 & - & - \\
\hline
\end{tabular}

${ }^{*}$ Defined as prednisolone use of $10 \mathrm{mg} /$ day (or equivalent) for more than 2 weeks during immunotherapy.

‡Requiring intravenous antibiotics.

Gender was excluded from the multivariate analysis due to its high correlation with smoking (Pearson's correlation: $p<0.001$ )
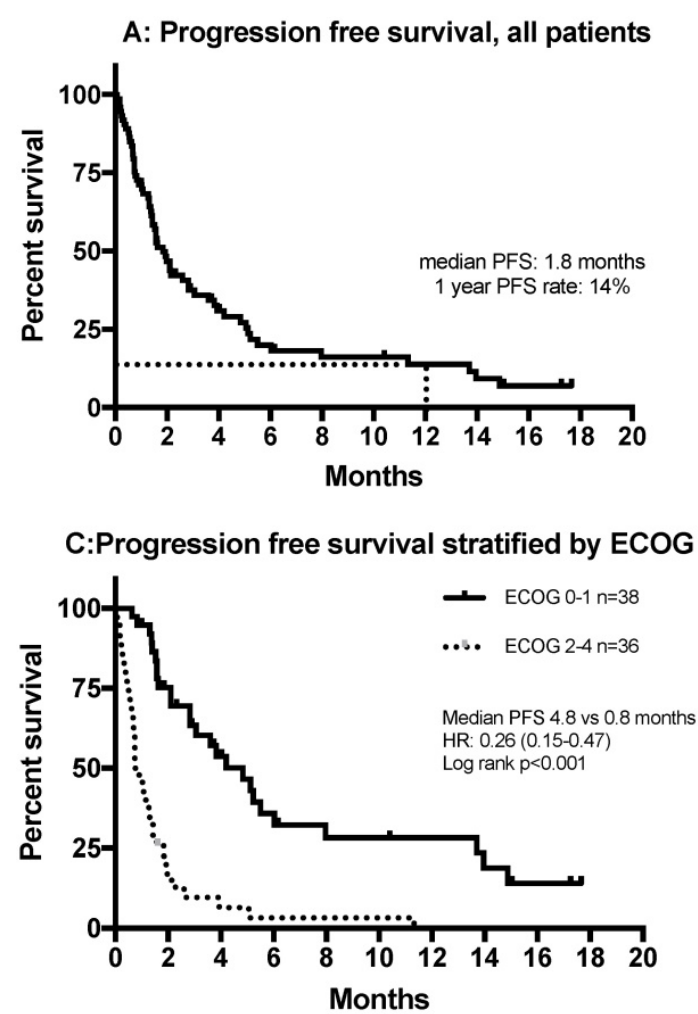

E: Progression free survival stratified by PD-L1

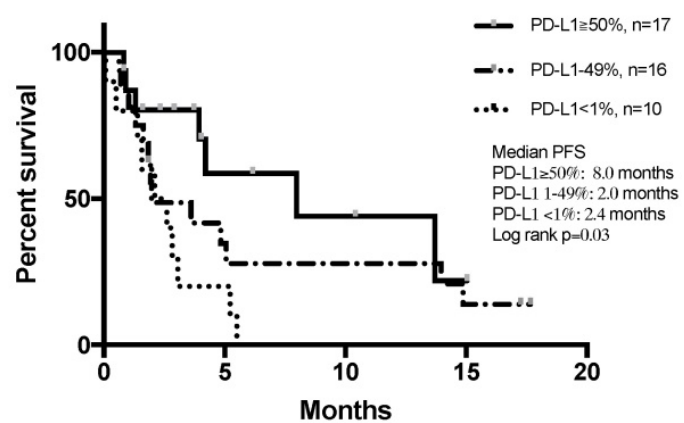

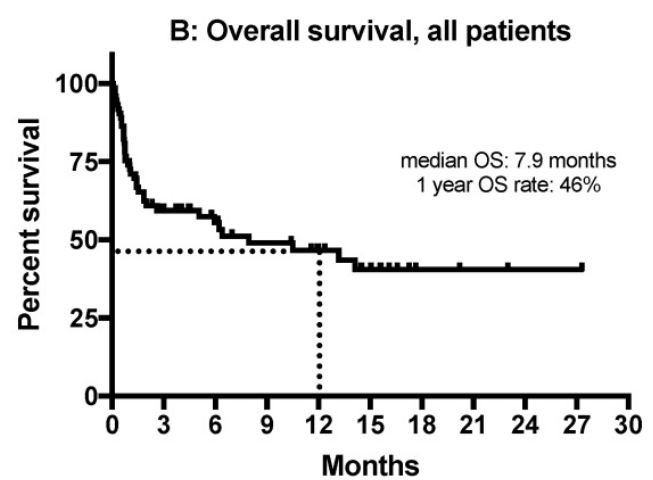

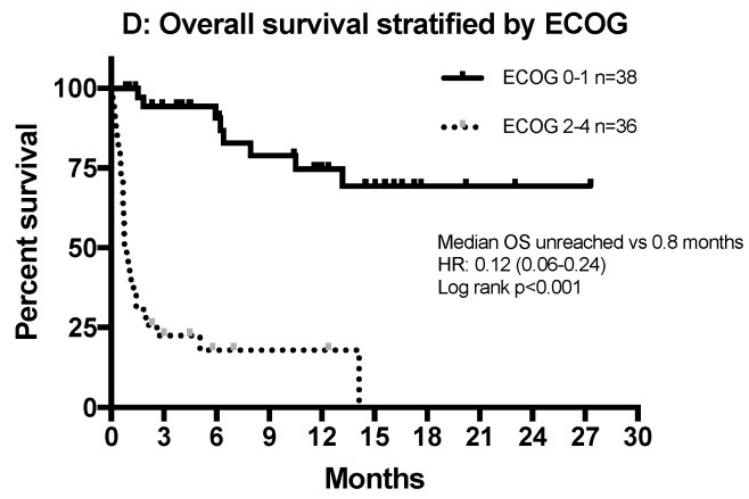

F: Overall survival stratified by PD-L1

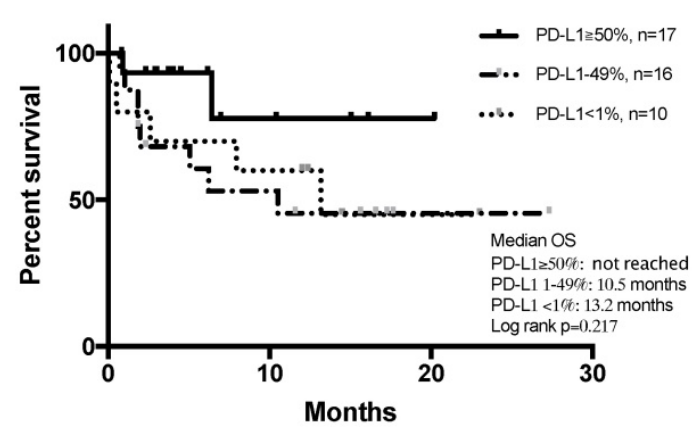

Figure 3: Kaplan-Meier curves of (A) PFS in all patients $(n=74)$; $(B)$ OS in all patients $(n=74)$; (C) PFS stratified by ECOG status; (D) OS stratified by ECOG status; (E) PFS stratified by PD-LI expression; (F) OS stratified by PD-LI expression. Abbreviations: ECOG: Eastern Cooperative Oncology Group Performance Status; HR, hazard ratio; OS, overall survival; PD-LI, programmed death-ligand 1; PFS, progression free survival. 


\section{Subsequent chemotherapy and PFS2}

Sixteen patients received subsequent chemotherapy after failure of immunotherapy, 7 with gemcitabine, 2 with docetaxel, 2 with vinorelbine, 1 with platinum-based doublet therapy, and 1 with cyclophosphamide/adriamycin/vincristine. Overall, these patients had an ORR of $23.1 \%$, a disease control rate (DCR) of $53.8 \%$ (Figure S2A), and a median PFS2 of 3.6 months (Figure S2B).

\section{Discussion}

This study reported the real-world effect of mono-immunotherapy with nivolumab or pembrolizumab for advanced-stage NSCLC using data from a large referral center in Taiwan. We demonstrated that the efficacy and safety profiles were similar with those reported in previous clinical trials[2, 3, 6, 14], even in patients who previously received intensive treatment. PD-L1 IHC staining via the 22C3 antibody correlated with favorable treatment response by trend, and EGFR mutation was a predictor of an unfavorable response. Smoking and ECOG performance status were significant and independent predictors of PFS and OS. These results imply that patients with poor ECOG status may be less likely to benefit from anti-PD-1 immunotherapy. The short median OS (0.8 months) of patients with poor ECOG status suggests that immunotherapy provides little or no benefit for these patients.

Previous phase 1 studies of nivolumab[14, 15] and pembrolizumab[6] examined patients with advanced-stage NSCLC who had already received multiple lines of therapy, and reported objective response rates of $19.4 \%$ to $23 \%$, median PFS of 3.6 to 3.7 months, and median OS of 12 to 19 months. Similarly, when we excluded patients with ECOG scores of 2 and above, the objective response rate was $34.2 \%$ and the median PFS was 4.8 months (although we did not attain a median OS). The main differences between the present study and the previous clinical trials are that we enrolled pure Eastern Asian patients, fewer of whom were smokers, and more of whom had EGFR mutations, characteristics that might indicate an unfavorable response to immunotherapy.[16, 17] Nonetheless, the clinical outcomes in our cohort (excluding patients with poor ECOG) were similar to those of the previous clinical trials. While current study enrolled heavily treated patients, there was also higher PD-L1 expression (PD-L1 $\geq 50 \%$ in our study, 38.6\%; PD-L1 $\geq 50 \%$ in Keynote 001, 23.3\%).[6] This could partly explain the similar outcome comparing with previous trials. PD-L1 expression had been reported to be higher in Asian comparing with Caucasians.[18] This means that anti-PD-1 immunotherapy for a real-life Eastern Asian population with advanced NSCLC may be as effective as reported in clinical trials.

There are few studies of the real-world experiences with anti-PD-1 treatment for patients with NSCLC. Brustugun and colleagues[7] examined a cohort of 58 Western NSCLC patients receiving nivolumab, and reported a median OS of 11.7 months. Most of the patients in that study had ECOG status of 2 and below (54/58, 93\%) and received immunotherapy as a first- or second-line treatment $(47 / 58$, $81 \%$ ). Another study by Diem and colleagues[8] examined a Western cohort of 52 NSCLC patients and reported median PFS and OS as 2.1 and 9.6 months, respectively. Similarly, most of the patients in this study had good ECOG status $(\leq 2,44 / 52,85 \%)$ and most received immunotherapy as a first- or second-line treatment $(31 / 54,60 \%)$. The survival rates in these previous studies were slightly better than in our cohort, suggesting that good performance status and earlier treatment improves survival for patients receiving anti-PD-1 immunotherapy.

Our data indicate that the presence of an EGFR mutation was associated with poor treatment response, similar to other clinical trials.[16] This implies that exclusion of patients with EGFR mutations will lead to a higher response rate to anti-PD-1 immunotherapy. Our results also suggest that PD-L1 status had a good correlation with response rate, and that these patients had a better PFS following anti-PD-1 treatment (although this was not statistically significant). Therefore, determination of PD-L1 status could be a convenient and inexpensive method to identify patients most likely to benefit from anti-PD-1 treatment in real-world settings, although its accuracy as a biomarker appears less than that of EGFR mutations in predicting the response to an EGFR tyrosine kinase inhibitor.[11, 19-22] KRAS mutation was a potential marker to predict better response to immunotherapy,[23, 24] our data had similar trend but was limited by small sample size.

Our results showed that smoking and ECOG status each had a significant and independent effect on PFS and OS. The effect of smoking might be because tobacco use leads to production of more non-synonymous mutations, and therefore generation of neoantigens that can potentiate the immune response to anti-PD-1 agents, [25] as reported similarly in previous studies. $[2,3,5,6,26]$ One of our important findings is that poor treatment outcome following anti-PD-1 therapy (median OS: 0.8 months) occurs in patients with poor ECOG scores, most of whom could not be evaluated. There may be two reasons why these drugs were given to patients in our study who had poor ECOG status. First, anti-PD-1 
immunotherapy generally has a lower toxicity than chemotherapy,[2-5] and might be considered tolerable even in very sick patients. Second, NSCLC patients with poor performance status have very limited choices of treatment, and family members may request immunotherapy as a last hope for survival, even when no strong evidence supports its use in these conditions. However, patients with poor ECOG status are usually immunocompromised, and have short life expectancy. Anti-PD-1 therapy must be given for a median period of 2 months to achieve benefits, and exhausted immunity may hamper treatment efficacy.[2, 3, 5, 6, 26, 27] Thus, our data suggest that use of anti-PD-1 treatment in NSCLC patients with poor ECOG status should be discouraged.

Some recent reports indicated improved efficacy of single-agent chemotherapy after failure of immunotherapy,[28-30] with an ORR ranging from $27 \%$ to $39 \%$. In our cohort, the ORR was $21.3 \%$, slightly better than reported for conventional single-agent salvage chemotherapy for lung cancer.[31] Preclinical studies suggested that certain chemotherapy agents may act through an immune-mediated mechanism,[32, 33] and some clinical trials suggest synergistic effects between anti-PD-1 immunotherapy and certain chemotherapy agents.[34] The effect of anti-PD-1 immunotherapy on the efficacy of subsequent chemotherapy needs confirmation in larger and more focused studies.

Previous clinical trials of anti-PD-1 monotherapy as a first- or second-line treatment indicated few toxicities.[2-6, 26] Diarrhea, pneumonitis, and hypothyroidism were the most common immunerelated adverse events. The current study also demonstrated a low rate of adverse events and few types of adverse events, with pneumonitis and hypothyroidism being the most common. This suggests that checkpoint blockade medications have a good safety profile, even among heavily treated and vulnerable patients (as in our cohort). Although rare, pneumonitis could necessitate interruption of immunotherapy, and should be closely monitored.

The study was limited by small patient numbers. We were forced to stratify patients into "evaluable" and "non-evaluable" groups as the non-evaluable patients could not have further tumor evaluation. This might lead to over-estimated response rate as those with rapid progression might be non-responders. However, the survival analysis would not be biased as we included the non-evaluable patients in the overall analysis.

In conclusion, we examined the real-world use of pembrolizumab and nivolumab on patients with advanced-stage NSCLC. Our cohort had a more heterogeneous background than patients in previous clinical trials. When either agent is given to patients with fair performance status (ECOG $\leq 1)$, the treatment outcomes were comparable those in previous trials, and the safety profile was acceptable. The use of PD-L1 IHC testing seems appropriate for providing guidance to clinicians regarding their selection of patients who may benefit most from anti-PD-1 therapy.

\section{Abbreviations}

CAV: cyclophosphamide Adriamycin and vincristine; DCR: disease control rate; ECOG: Eastern Cooperative Oncology Group Performance Status; EGFR: epidermal growth factor receptor; IHC: Immunohistochemical staining; KRAS: Kirsten rat sarcoma virus oncogene homolog; NSCLC: non-small cell lung cancer; OS: overall survival; ORR: objective response rate; PD-1: programmed death 1; PD: progression of disease; PFS: progression free survival; PR: partial response; RECIST: Response Evaluation Criteria In Solid Tumors; SCLC: small cell lung cancer; SD: stable disease.

\section{Acknowledgements}

All authors read and approved the final manuscript. We thank all the patients and their families and caregivers for participating in this study.

\section{Supplementary Material}

Supplementary figures and tables.

http://www.jcancer.org/v09p1813s1.pdf

\section{Conflicts of Interest}

Jin-Yuan Shih received speaking honoraria from AstraZeneca, Roche, Boehringer Ingelheim, Pfizer, Norvatis, Bristol-Myers Squibb, Merck Sharp \& Dohme and Eli Lilly, and has been paid for a consulting or advisory role by AstraZeneca, Roche, Boehringer Ingelheim, Novartis, Merck Sharp \& Dohme, AbbVie and Chugai Pharmaceutical. Other authors declared no conflicts of interest. James Chih-Hsin Yang is a member of the advisory committees and has received honoraria from AstraZeneca, Roche/Genentech, Boehringer Ingelheim, MSD, Merck Serono, Novartis, Pfizer, Clovis Oncology, Eli Lilly, Bayer, Celgene, Astellas, BMS, Ono Pharmaceutical, Yuhan Pharmaceutical, and Chugai Pharmaceutical.

\section{References}

1. Mountzios G, Linardou H, Kosmidis P. Immunotherapy in non-small cell lung cancer: the clinical impact of immune response and targeting. Ann Transl Med. 2016; 4: 268.

2. Borghaei H, Paz-Ares L, Horn L, Spigel DR, Steins M, Ready NE, et al. Nivolumab versus Docetaxel in Advanced Nonsquamous Non-Small-Cell Lung Cancer. New England Journal of Medicine. 2015; 373: 1627-39. 
3. Brahmer J, Reckamp KL, Baas P, Crinò L, Eberhardt WEE, Poddubskaya E, et al. Nivolumab versus Docetaxel in Advanced Squamous-Cell Non-Small-Cell Lung Cancer. New England Journal of Medicine. 2015; 373: 123-35.

4. Herbst RS, Baas P, Kim D-W, Felip E, Pérez-Gracia JL, Han J-Y, et al. Pembrolizumab versus docetaxel for previously treated, PD-L1-positive, advanced non-small-cell lung cancer (KEYNOTE-010): a randomised controlled trial. The Lancet. 2016; 387: 1540-50.

5. Reck M, Rodríguez-Abreu D, Robinson AG, Hui R, Csószi T, Fülöp A, et al. Pembrolizumab versus Chemotherapy for PD-L1-Positive Non-Small-Cell Lung Cancer. New England Journal of Medicine. 2016; 75: 1823-33.

6. Garon EB, Rizvi NA, Hui R, Leighl N, Balmanoukian AS, Eder JP, et al. Pembrolizumab for the Treatment of Non-Small-Cell Lung Cancer. New England Journal of Medicine. 2015; 372: 2018-28.

7. Brustugun OT, Sprauten M, Helland A. Real-world data on nivolumab treatment of non-small cell lung cancer. Acta Oncologica. 2017; 56: 438-40.

8. Diem S, Schmid S, Krapf M, Flatz L, Born D, Jochum W, et al. Neutrophil-to-Lymphocyte ratio (NLR) and Platelet-to-Lymphocyte ratio (PLR) as prognostic markers in patients with non-small cell lung cancer (NSCLC) treated with nivolumab. Lung Cancer. 2017; 111: 176-81.

9. Maemondo M, Inoue A, Kobayashi K, Sugawara S, Oizumi S, Isobe H, et al. Gefitinib or Chemotherapy for Non-Small-Cell Lung Cancer with Mutated EGFR. New England Journal of Medicine. 2010; 362: 2380-8.

10. O'Connor J, Seidl-Rathkopf K, You P, Nussbaum NC, Torres AZ, Fessele KL, et al. Adoption of immunotherapy into real-world practice: Insights from the use of checkpoint inhibitors. Journal of Clinical Oncology. 2017; 35(e):14583.

11. Mok TS, Wu YL, Thongprasert S, Yang CH, Chu DT, Saijo N, et al. Gefitinib or carboplatin-paclitaxel in pulmonary adenocarcinoma. The New England journal of medicine. 2009; 361: 947-57.

12. Hsu KH, Ho CC, Hsia TC, Tseng JS, Su KY, Wu MF, et al. Identification of five driver gene mutations in patients with treatment-naive lung adenocarcinoma in Taiwan. PLOS ONE. 2015; 10: e0120852.

13. Su KY, Kao JT, Ho BC, Chen HY, Chang GC, Ho CC, et al. Implementation and Quality Control of Lung Cancer EGFR Genetic Testing by MALDI-TOF Mass Spectrometry in Taiwan Clinical Practice. Sci Rep. 2016; 6: 30944

14. Gettinger SN, Horn L, Gandhi L, Spigel DR, Antonia SJ, Rizvi NA, et al. Overall Survival and Long-Term Safety of Nivolumab (Anti-Programmed Death 1 Antibody, BMS-936558, ONO-4538) in Patients With Previously Treated Advanced Non-Small-Cell Lung Cancer. Journal of Clinical Oncology. 2015; 33: 2004-12.

15. Gettinger S, Rizvi NA, Chow LQ, Borghaei H, Brahmer J, Ready N, et al. Nivolumab Monotherapy for First-Line Treatment of Advanced Non-Small-Cell Lung Cancer. Journal of Clinical Oncology. 2016; 34: 2980-7.

16. Lee CK, Man J, Lord S, Links M, Gebski V, Mok T, et al. Checkpoint Inhibitors in Metastatic EGFR-Mutated Non-Small Cell Lung Cancer-A Meta-Analysis. J Thorac Oncol. 2017; 12: 403-7.

17. Rizvi NA, Hellmann MD, Snyder A, Kvistborg P, Makarov V, Havel JJ, et al. Cancer immunology. Mutational landscape determines sensitivity to PD-1 blockade in non-small cell lung cancer. Science. 2015; 348: 124-8.

18. Lin $\mathrm{Z}, \mathrm{Xu} \mathrm{Y,} \mathrm{Zhang} \mathrm{Y}, \mathrm{He} \mathrm{Q}$, Zhang J, He J, et al. The prevalence and clinicopathological features of programmed death-ligand 1 (PD-L1) expression: a pooled analysis of literatures. Oncotarget. 2016; 7: 15033-46.

19. Maemondo M, Inoue A, Kobayashi K, Sugawara S, Oizumi S, Isobe H, et al. Gefitinib or chemotherapy for non-small-cell lung cancer with mutated EGFR. N Engl J Med. 2010; 362: 2380-8.

20. Mitsudomi T, Morita S, Yatabe Y, Negoro S, Okamoto I, Tsurutani J, et al. Gefitinib versus cisplatin plus docetaxel in patients with non-small-cell lung cancer harbouring mutations of the epidermal growth factor receptor (WJTOG3405): an open label, randomised phase 3 trial. Lancet Oncol. 2010; 11: $121-8$

21. Rosell R, Carcereny E, Gervais R, Vergnenegre A, Massuti B, Felip E, et al. Erlotinib versus standard chemotherapy as first-line treatment for European patients with advanced EGFR mutation-positive non-small-cell lung cancer (EURTAC): a multicentre, open-label, randomised phase 3 trial. Lancet Oncol. 2012; 13: 239-46.

22. Zhou $\mathrm{C}$, Wu YL, Chen G, Feng J, Liu XQ, Wang $C$, et al. Erlotinib versus chemotherapy as first-line treatment for patients with advanced EGFR mutation-positive non-small-cell lung cancer (OPTIMAL, CTONG-0802): a multicentre, open-label, randomised, phase 3 study. Lancet Oncol. 2011; 12: $735-42$

23. Dong ZY, Zhong WZ, Zhang XC, Su J, Xie Z, Liu SY, et al. Potential Predictive Value of TP53 and KRAS Mutation Status for Response to PD-1 Blockade Immunotherapy in Lung Adenocarcinoma. Clin Cancer Res. 2017; 23: 3012-24.

24. Kim JH, Kim HS, Kim BJ. Prognostic value of KRAS mutation in advanced non-small-cell lung cancer treated with immune checkpoint inhibitors: A meta-analysis and review. Oncotarget. 2017; 8: 48248-52.

25. Rizvi NA, Hellmann MD, Snyder A, Kvistborg P, Makarov V, Havel JJ, et al. Mutational landscape determines sensitivity to PD-1 blockade in non-small cell lung cancer. Science. 2015; 348: 124-8.

26. Carbone DP, Reck M, Paz-Ares L, Creelan B, Horn L, Steins M, et al. First-Line Nivolumab in Stage IV or Recurrent Non-Small-Cell Lung Cancer. New England Journal of Medicine. 2017; 376: 2415-26.

27. Blackburn SD, Shin H, Freeman GJ, Wherry EJ. Selective expansion of a subset of exhausted CD8 T cells by alphaPD-L1 blockade. Proceedings of the National Academy of Sciences of the United States of America. 2008; 105: 15016-21.
28. Grigg C, Reuland BD, Sacher AG, Yeh R, Rizvi NA, Shu CA. Clinical outcomes of patients with non-small cell lung cancer (NSCLC) receiving chemotherapy after immune checkpoint blockade. Journal of Clinical Oncology. 2017; 35: 9082

29. Schvartsman G, Peng SA, Bis G, Lee JJ, Benveniste MFK, Zhang J, et al. Response to single-agent (SA) chemotherapy (CTx) after immunotherapy exposure in non-small cell lung cancer (NSCLC). Journal of Clinical Oncology. 2017; 35: 9083.

30. Leger PD, Rothschild S, Castellanos E, Pillai RN, York SJ, Horn L. Response to salvage chemotherapy following exposure to immune checkpoint inhibitors in patients with non-small cell lung cancer. Journal of Clinical Oncology. 2017; 35: 9084.

31. Shepherd FA, Dancey J, Ramlau R, Mattson K, Gralla R, O'Rourke M, et al. Prospective Randomized Trial of Docetaxel Versus Best Supportive Care in Patients With Non-Small-Cell Lung Cancer Previously Treated With Platinum-Based Chemotherapy. Journal of Clinical Oncology. 2000; 18: 2095-103.

32. Gebremeskel S, Lobert L, Tanner K, Walker B, Oliphant T, Clarke LE, et al. Natural killer T-cell immunotherapy in combination with chemotherapy-induced immunogenic cell death targets metastatic breast cancer. Cancer Immunol Res. 2017.

33. Cui S. Immunogenic Chemotherapy Sensitizes Renal Cancer to Immune Checkpoint Blockade Therapy in Preclinical Models. Med Sci Monit. 2017; 23: 3360-6.

34. Langer CI, Gadgeel SM, Borghaei H, Papadimitrakopoulou VA, Patnaik A, Powell SF, et al. Carboplatin and pemetrexed with or without pembrolizumab for advanced, non-squamous non-small-cell lung cancer: a randomised, phase 2 cohort of the open-label KEYNOTE-021 study. Lancet Oncol. 2016; 17: 1497-508. 